\title{
Effect of Airfoil and Composite Layer Thicknesses on an Aerostructural Blade Optimization for Wind Turbines
}

\author{
Ryan Barrett \\ Brigham Young University, ryan.barrett100@gmail.com \\ Ian Freeman \\ Brigham Young University, ianj.freeman@gmail.com \\ Andrew Ning \\ Brigham Young University, aning@byu.edu
}

Follow this and additional works at: https://scholarsarchive.byu.edu/facpub

Part of the Mechanical Engineering Commons

\section{Original Publication Citation}

Barrett, R., Freeman, I., and Ning, A., "Effect of Airfoil and Composite Layer Thicknesses on an Aerostructural Blade Optimization for Wind Turbines," IEEE Conference on Technologies for Sustainability, Ogden, UT, Jul. 2015. doi:10.1109/SusTech.2015.7314346

\section{BYU ScholarsArchive Citation}

Barrett, Ryan; Freeman, lan; and Ning, Andrew, "Effect of Airfoil and Composite Layer Thicknesses on an Aerostructural Blade Optimization for Wind Turbines" (2015). Faculty Publications. 1722.

https://scholarsarchive.byu.edu/facpub/1722 


\title{
Effect of Airfoil and Composite Layer Thicknesses on an Aerostructural Blade Optimization for Wind Turbines
}

\author{
Ryan Barrett, Ian Freeman, and Andrew Ning \\ Mechanical Engineering Department \\ Brigham Young University \\ Provo, Utah 84602 \\ Email: ryan.t.barrett@byu.edu
}

\begin{abstract}
The purpose of this research is to enhance the performance of wind turbine blades by exploring the effect of adding airfoil and material layer thicknesses to the optimization design process. This is accomplished by performing an aerostructural blade optimization to minimize mass over annual energy production and thereby reduce the cost of energy. Changing airfoil thickness allows the airfoil shape to evolve as part of the optimization. The airfoil thicknesses are allowed to vary within two airfoil families, the TU-Delft and NACA 64-series, that are used in the NREL 5-MW reference turbine. Both experimental wind tunnel and computational data are used to estimate the blade's aerodynamic performance. Material layer thicknesses in the composite lamina spar cap and trailing edge panels are separated and added to the optimization. Results show a reduction of $0.8 \%$ in the full optimization as compared to the reference turbine due to an increase in energy production $(+0.6 \%)$ and decrease in blade mass $(-2.7 \%)$.
\end{abstract}

Keywords - wind turbines, blade optimization, aerostructural, free-form, XFOIL, composite, airfoil thickness

\section{NOMENCLATURE}

$\begin{array}{ll}C O E & \text { cost of energy } \\ A E P & \text { annual energy production } \\ t / c & \text { airfoil thickness to chord ratio } \\ c_{l} & \text { coefficient of lift } \\ c_{d} & \text { coefficient of drag } \\ \alpha & \text { angle of attack } \\ m / A E P & \text { mass over annual energy production } \\ T R I A X & \text { tri-axial structural composite layer }\end{array}$

\section{INTRODUCTION}

Wind turbines are a growing source of renewable energy that is important across many fields such as engineering, business, and politics. Through the use of optimization, wind turbine performance can be improved and enhanced. While wind turbine optimization can be quite broad in scope, this research focuses on the wind turbine blade. A wind turbine blade has three main objectives: extract energy from the wind through its aerodynamics performance, withstand the loads, stresses, and strains that are present during operation, and maintain a low cost of energy compared to other energy sources. An aerostructural optimization helps to achieve these objectives. The problem, however, is that blade optimization is often done sequentially, where airfoil shapes that make up the blade are chosen beforehand and then the blade is optimized [1]. Fixing the shape of the airfoils during the blade optimization process can be seen as a limitation to finding a more optimal blade design. Similarly, the thicknesses of the materials that make up the blade structure are often either left fixed or all the materials thicknesses are combined to be optimized as a single thickness. This research seeks to explore how adding airfoil thickness and the individual material layer thicknesses in the blade's composite lamina sections contribute to reducing the cost of energy, making wind energy a more attractive alternative energy source.

Conventional blade optimization consists of choosing several airfoil shapes across the blade span that remain fixed throughout the optimization as shown in Fig. 1. One weakness in this approach is that it fails to fully capture the trade-offs that exist between the aerodynamic performance and the structural integrity of the wind turbine blade. The airfoil shape has not been generally added to blade optimization in the past due to its complexity. Including the airfoil shape adds many design degrees of freedom and more closely couples the blade's aerodynamics and structure that can often be easier to design separately. In addition, experimental wind tunnel data is often not available for many airfoil shapes and computational panel methods have a tendency to be not very accurate and not converge in separated flow. However, a free-form approach has 
been used in which the entire airfoil shape can change as part of the optimization. Used often in aircraft wing design, this approach has a growing presence in wind turbine blade design. A number of parameters are used to define the airfoil shape and these parameters are treated as design variables in the optimization process.

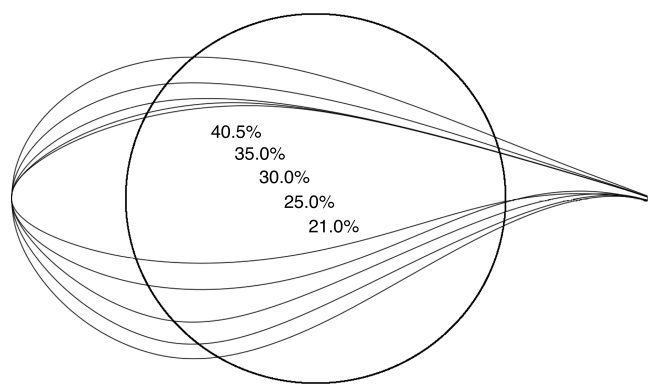

Fig. 1: Conventional approach to blade optimization with fixed airfoil thickness to chord ratios (blade side view)

The ability to evolve and adapt the airfoil shape within the optimization can better extract the most optimal blade performance and lead to improved solutions over existing designs that use fixed airfoil shapes. The advantage of this free-form approach becomes apparent for applications where blade performance is more sensitive to changes in blade thickness. A free-form aerostructural optimization has been shown by Bottasso et al. [2] to decrease $C O E$ (cost of energy) in low induction rotors. Another potential application where this becomes meaningful is in turbines with high tip-speeds. The $C O E$ is more responsive to small variances in blade thickness in these high tip-speed turbines than in normal wind turbines [3]. A main advantage of increased tip speed is the reduction of peak torque loads. This aids significantly in the structural optimization as it allows for a substantial size reduction in drivetrain mass and costs [4]. Efficient high tip-speed designs have been shown to boost performance by as much as $25-30 \%$ by increasing the speed and thereby generating more power and lowering drivetrain mass [5].

Despite the advantages of this free-form approach, the implementation in optimization can become quite complex with the addition of many different design variables. By pre-selecting the airfoil family, the complexity of the free-form method can be reduced to a single parameter: the airfoil thickness. A precomputed spline is used to provide continuous and differentiable data for all airfoil thickness to chord ratios $(t / c)$ within specified ranges of interest. The spline is anchored with experimental wind tunnel data and augmented with computational data. Including $t / c$ as a design variable can result in greater blade performance [6].

Similar to the airfoil thickness, the material layers thicknesses in the structural composite lamina sections are often left fixed or optimized as a single design variable. This research expands the optimization of the material layers by separating the material thicknesses in the composite lamina section in both the spar cap and the trailing edge panels. Doing so provides the flexibility to change each material thickness as needed for the specific design problem.

The desired result of this research is to better explore the effect of adding in airfoil and material layer thicknesses compared to the conventional approach. While $C O E$ is the ultimate goal of wind turbine optimization, optimizing for $m / A E P$ (the total turbine mass over annual energy production) approximates $C O E$ as shown by Ning et al. [7] for fixed diameter rotors. By fixing the rotor diameter and rotor thrust and including the total turbine mass (including the blades, hub, nacelle, and tower), minimizing $m / A E P$ becomes a valid approximation for minimizing $C O E$. As $C O E$ is a measure of the total system cost divided by the annual energy production, $m / A E P$ proves to be a valid comparison because the mass of the wind turbine is in part proportional to its cost. The reduction in $m / A E P$ is achieved by increasing $A E P$ and reducing mass. The blade aerodynamics are affected by the airfoil thickness, chord, twist, and tip-speed ratio while the blade structure is affected by varying the thicknesses of the different material layer thicknesses in the trailing edge and spar cap. By adding the airfoil and material layer thicknesses the cost of wind energy can be reduced.

\section{Methodology}

To achieve the desired objective, the airfoil and material layer thicknesses had to be prepared to be continuous and differentiable for effective use of gradient-based optimization techniques. This process is described in more detail below.

\section{A. Aerodynamics - Airfoil Thicknesses}

For the airfoil thicknesses to be continuous and differentiable, the lift and drag coefficients $\left(c_{l}\right.$ and $c_{d}$ ) needed to be estimated as a function of any angle of attack $(\alpha)$ and $t / c$. This was accomplished with a combination of wind tunnel and computational $c_{l}$ and $c_{d}$ aerodynamic data for the TU-Delft and the NACA 64-series family of airfoils. These families were chosen because they are used in the NREL 5-MW reference turbine and wind tunnel data is available. The TU-Delft 
family has available wind tunnel data for the following airfoil thickness to chord ratios: $40.5 \%, 35.0 \%, 30.0 \%$, $25.0 \%$, and $21.0 \%$. Wind tunnel data is also available for the NACA 64 -series at $18 \%$. To more closely match the NREL 5-MW three-bladed reference turbine, the TU-Delft airfoil family was used for the first two-thirds of the blade span and the NACA 64-series airfoils for the final third of the blade span.

XFOIL, which is a software program that can be used in the design and analysis of subsonic isolated airfoils [8], was used to perform the aerodynamic computational analysis for the thinner airfoils where wind tunnel data was not available: $15 \%$ to $21 \%$ for the TU-Delft family of airfoils and $12 \%$ to $21 \%$ for the NACA 64 -series airfoils. The XFOIL analysis was performed with varying thicknesses for angles of attack from $-20^{\circ}$ to $20^{\circ}$ with a Reynolds number of $10^{6}$ and $N_{\text {crit }}$ of 9 (corresponding to the turbulence of an average wind tunnel). Rotational corrected and extrapolated data was obtained using the NREL AirfoilPrep Python tool [9]. The 3D rotational corrections were performed using Du's method to augment the lift and Eggers' method to modify the drag. The Viterna method was used to extrapolate the lift and drag coefficients from $-180^{\circ}$ to $180^{\circ}$. The computational data gathered from XFOIL was only used for modest airfoil thicknesses $(t / c$ less than 21\%) and modest angles of attack $\left(-20^{\circ}\right.$ to $\left.20^{\circ}\right)$ as XFOIL has known issues such as a lack of convergence in highly separated flows.

The combination of the wind tunnel and XFOIL data is justified based on the aspiration for higher fidelity results and the relatively close approximation between the two at the point of intersection (at $t / c$ $=21 \%$ ) as seen in Fig. 2. A correction had to be made to the XFOIL data, however, to increase the accuracy to match the wind tunnel data. The lift and drag coefficients from the wind tunnel and XFOIL were compared for known thicknesses. The average difference for each $t / c$ at each angle of attack for both the lift and drag coefficients was applied to the XFOIL data where wind tunnel data was not available. As XFOIL is based on idealized computational models, it tends to under-predict drag coefficients and slightly over-predict lift coefficients. Therefore, the XFOIL correction is a drag and lift offset based on the known difference between the wind tunnel and computational data under the same conditions.

A 2D spline interpolation of the lift and drag coefficients allow the $c_{l}$ and $c_{d}$ to be estimated for any $\alpha$ and $t / c$ within the specified bounds for both airfoil families. A smoothing factor of 0.001 and 0.0005 was made to the lift and drag coefficient splines, respectively. The smoothing factors are a similar order of magnitude for both the lift and drag coefficients. Seven thicknesses (five wind tunnel and two XFOIL) were used for the creation of the TU-Delft surface and four (one wind tunnel and three XFOIL) for the creation of the NACA 64-series surface. These response surfaces can be seen in Fig. 3. This figure demonstrates the ability to analyze the performance of an airfoil for any $\alpha$ and $t / c$ as well as to being continuously differentiable everywhere in the range, which is essential to gradient-based optimization.

The lift over drag ratio as a function of $t / c$ is shown in Fig. 4 and compares the original XFOIL, corrected XFOIL, and wind tunnel data to the spline with the smoothing factor applied. The lift to drag ratio is an important metric in analyzing the aerodynamic performance of the blade. The aerodynamic blade analysis was performed using a blade element momentum method with guaranteed convergence (CCBlade) [10] using the NREL wind blade analysis tool RotorSE [11]. Standard IEC specifications for a land-based high-wind-speed site (IEC Class IB) was used corresponding to a mean wind speed of 10.0 $\mathrm{m} / \mathrm{s}$ [12]. The wind conditions followed a Weibull distribution with a shape parameter of 2.0.

\section{B. Structures - Material Layer Thicknesses}

The structural aspect consisted of changing the material layer thicknesses of different composite lamina sections of the blade. The focus was on sector 2, the spar cap panel, and sector 3, the trailing edge panel, as seen in Fig. 5. There are a number of layers including: the GelCoat, glass fabrics, SNL TRIAX ([ \pm 45$] 2[0] 2)$, SaerTex Double-Dias (DB, $[ \pm 45] 4)$, carbon fabrics, generic foam, and epoxy resins [7]. This research focuses on three of the different layers: the SNL TRIAX, carbon fabrics, and generic foam layers. The SNL TRIAX (triaxial) is a composite layer that consists of glass fiber reinforced plastic (GFRP) and carbon fiber reinforced plastic (CFRP). The spar cap consists of TRIAX and carbon layers while the trailing edge consists of TRIAX and foam layers. The thicknesses of these material layers are added to the structural optimization. In the conventional approach, these thicknesses are either not added at all or the thickness of all the layers is added as a single design variable. This single design variable approach, however, only scales the different layers linearly. By separating the layers, the proportion of each material layer can vary so as to find the optimal thickness. 


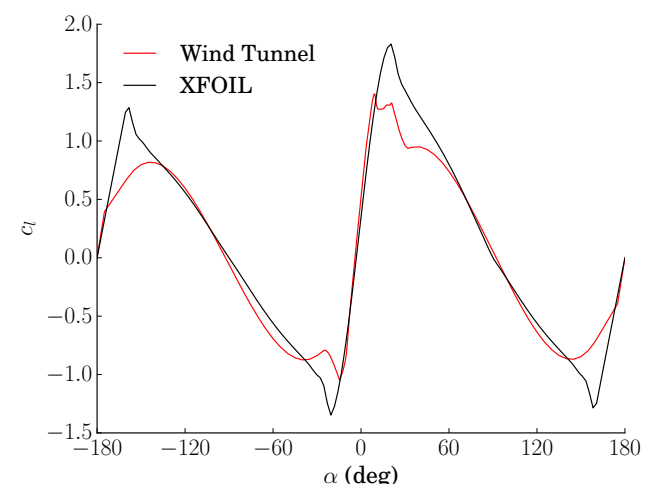

(a) $c_{l}$ versus $\alpha$

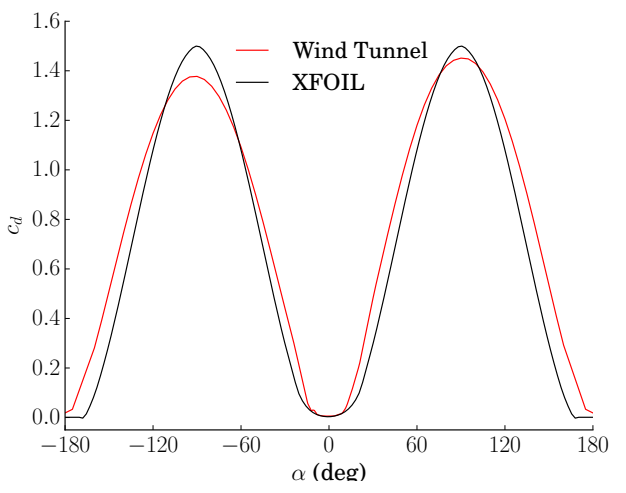

(b) $c_{d}$ versus $\alpha$

Fig. 2: Rotational corrected and extrapolated XFOIL compared to wind tunnel data for TU-Delft $t / c=21 \%$

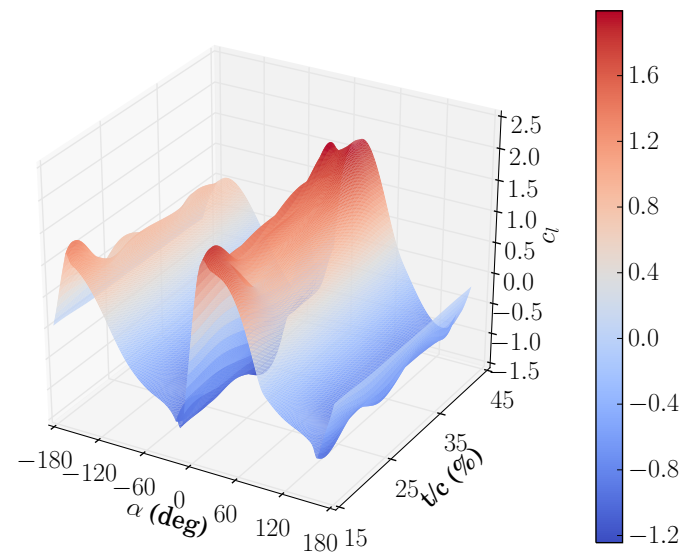

(a) $c_{l}$ surface used for airfoil thickness optimization

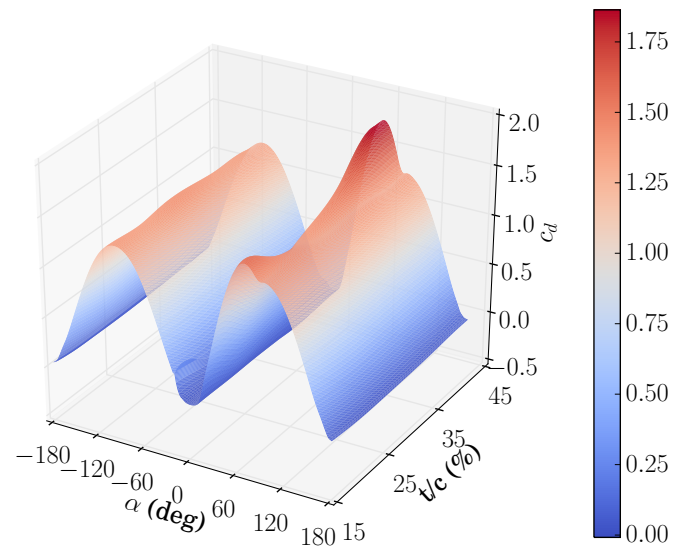

(b) $c_{d}$ surface used for airfoil thickness optimization

Fig. 3: Comparison of the $c_{l}$ and $c_{d}$ surfaces for TU-Delft airfoils

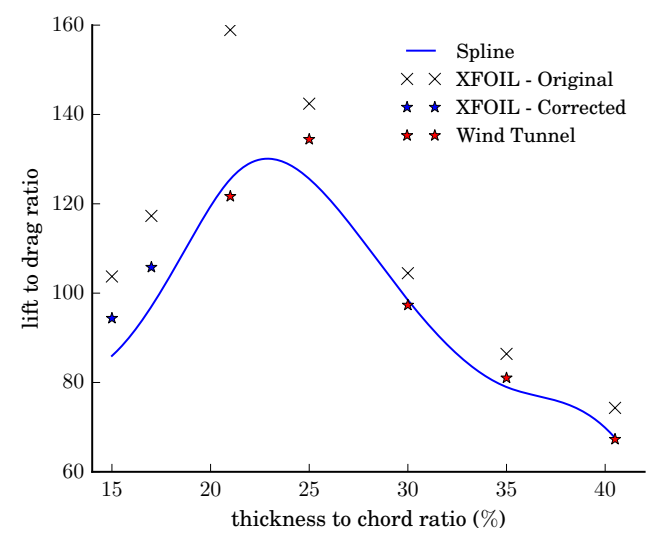

(a) TU-Delft airfoil family

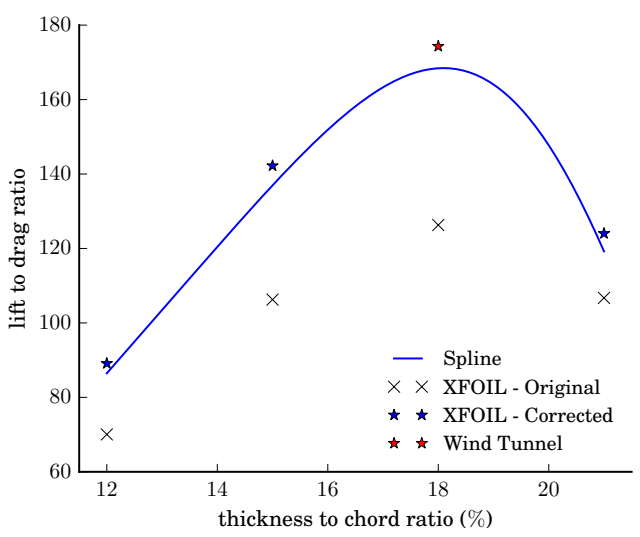

(b) NACA 64-series airfoils

Fig. 4: Lift to drag ratio curves for $\alpha=5^{\circ}$ 


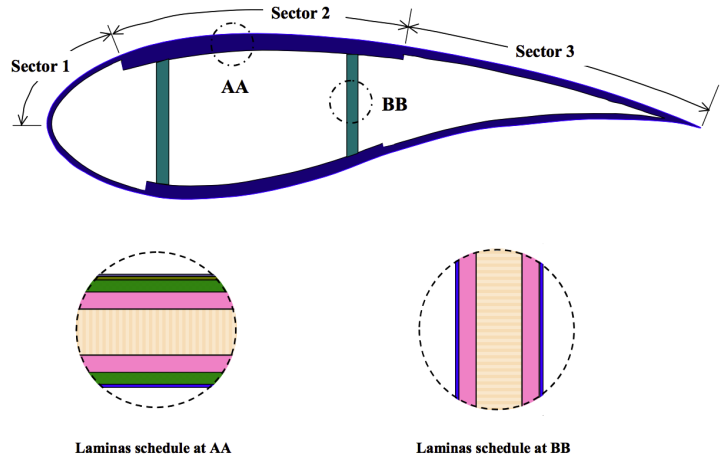

Fig. 5: Composite layers for a wind turbine blade. Figure from Bir et al. [13]

The material thicknesses for the spar cap and trailing edge panel are independently defined using Akima splines. There are five control points used to control the thicknesses at thirty eight points along the blade. These parameters, along with relevant material properties, are used to construct and evaluate a finite element analysis model of the blade, while the control points for these splines are used as design variables. The outputs from the structural analysis inform both the objective and the majority of the constraints for the model.

\section{Optimization}

The optimization objective function is to minimize $m / A E P$. All the relevant structural and aerodynamic design variables are combined into a single optimization problem. The design variables consist of nine main categories, five of which are aerodynamic design variables and four of which are structural design variables. Several of these variables are arrays that define control points on a spline. Three aerodynamic (airfoil thickness to chord ratio, chord, and twist) and all of the structural design variables (spar cap TRIAX, spar cap carbon, trailing edge TRIAX, and trailing edge foam thicknesses) control splines that define the entire blade. For $t / c$, the first five points control the TU-Delft and the last one controls the NACA 64-series airfoils. The design variables are summarized in Table I. In total, there are 36 design variables used in the optimization.

There are a number of structural constraints on the optimization that are grouped into six main categories. These categories include constraints on the strain and buckling of the spar cap and trailing edge, the flap-wise and edge-wise frequency, and the rotor thrust. These are summarized in Table II.
TABLE I: Design Variables

\begin{tabular}{lll}
\hline Description & & \# of vars. \\
\hline airfoil thickness to chord ratio distribution & $t / c_{i}$ & 6 \\
chord distribution & $c_{i}$ & 4 \\
max chord location & $\mathrm{r}_{i} \mathrm{c}$ & 1 \\
twist distribution & $\theta_{i}$ & 4 \\
tip-speed ratio & $\lambda$ & 1 \\
spar cap - TRIAX thickness distribution & tspart $_{i}$ & 5 \\
spar cap - carbon thickness distribution & tsparc $_{i}$ & 5 \\
trailing edge - TRIAX thickness distribution & ttept $_{i}$ & 5 \\
trailing edge - foam thickness distribution & ttepf $_{i}$ & 5 \\
\hline
\end{tabular}

TABLE II: Constraints

\begin{tabular}{l}
\hline Description \\
\hline spar cap strain $\leq$ ultimate strain at 7 stations along blade \\
trailing edge strain $\leq$ ultimate strain at 8 stations along blade \\
spar cap buckling $\leq$ critical buckling at 8 stations along blade \\
trailing edge buckling $\leq$ critical buckling at 7 stations along blade \\
flap-wise/edge-wise frequency $\geq$ blade passing frequency \\
rotor thrust $\leq$ initial rotor thrust \\
\hline
\end{tabular}

The strain is constrained for extreme load conditions according to IEC standards. The buckling is constrained for maximum operating conditions. All natural frequencies had to be above the blade natural frequency with an added margin to avoid resonance. While only the mass of the blades could change as part of the optimization, the calculation of the $m / A E P$ included the entire system mass for the cost of energy to be best approximated. For the $m / A E P$ approximation to be valid the rotor thrust had to be fixed to conservatively neglect the impact on the tower and the drivetrain mass. The rated power was kept constant at 5-MW for a similar reason. Additional detail on these constraints is described by Ning et al. [7]. The final optimization is summarized below:

$$
\begin{array}{ll}
\text { minimize } & m / A E P \\
\text { with respect to } & \mathrm{x}=t / c_{i}, c_{i}, \theta_{i}, \lambda, r_{c}, \text { tspart }_{i}, \\
& \text { tsparc }_{i}, \text { ttept }_{i}, \text { ttep }_{i} \\
\text { subject to } & \text { buckling, strain, natural frequency, rotor thrust }
\end{array}
$$

The optimization was performed using a gradient-based sequential quadratic programming method using the SNOPT [14] optimization package within the OpenMDAO [15] framework for multidisciplinary optimization. The main challenges in performing the gradientbased optimization were in scaling and obtaining gradients. Gradients were obtained through a combination of analytic and finite difference gradients as RotorSE already has many analytic gradients built in, and finite differencing was used to provide the remaining gradients in the OpenMDAO framework. Both the objective and some of the design variables, such as the airfoil and material thicknesses thicknesses, were scaled to be of a similar order of magnitude. 
TABLE III: Optimization Results

\begin{tabular}{|c|c|c|c|c|c|}
\hline & units & Reference Blade & Conventional & Airfoil Thickness Fixed & Full Optimization \\
\hline$c_{i}$ & $\mathrm{~m}$ & {$[3.26,4.57,3.32,1.46]$} & {$[2.19,4.85,3.34,1.79]$} & {$[2.12,4.95,3,33,1.82]$} & {$[3.30,4.47,3.40,1.70]$} \\
\hline$\theta_{i}$ & $\theta$ & {$[13.28,7.46,2.89,-0.09]$} & {$[15.15,5.84,1.38,-0.60]$} & {$[14.01,5.65,1.06,-0.60]$} & {$[13.36,7.34,2.53,-0.17]$} \\
\hline$\lambda$ & & 7.55 & 7.98 & 8.12 & 7.75 \\
\hline$r_{c} \mathrm{c}$ & & 0.236 & 0.144 & 0.125 & 0.201 \\
\hline tspart $_{i}$ & $\mathrm{~mm}$ & {$[3.0,2.9,2.8,2.75,2.7]$} & - & {$\left[\begin{array}{lllll}1.2 & 1.2 & 1.2 & 1.2 & 1.2\end{array}\right]$} & {$[2.48,2.41,2.34,2.30,2.26]$} \\
\hline tsparc $_{i}$ & $\mathrm{~mm}$ & {$[42,25,10,9,6.6]$} & - & {$[43.2,30.7,19.8,17.87 .9]$} & {$[42,25.1,10.2,9.3,6.6]$} \\
\hline ttept $_{i}$ & $\mathrm{~mm}$ & {$[3.0,2.9,2.8,2.75,2.7]$} & - & {$\left[\begin{array}{lllll}1.2 & 1.2 & 1.2 & 1.2 & 1.2\end{array}\right]$} & {$[2.48,2.41,2.34,2.30,2.26]$} \\
\hline ttepf $_{i}$ & $\mathrm{~mm}$ & {$[90,70,50,30,20]$} & - & {$[91,73.4,51.9,31.8,20.2]$} & {$[90.1,70.2,50.2,30.1,20]$} \\
\hline$t / c_{i}$ & $\%$ & $\begin{array}{l}{[40.5,35.0,30.0} \\
25.0,21.0,18.0]\end{array}$ & - & - & $\begin{array}{l}{[33.1,29.1,25.4} \\
18.8,16.7,15.2]\end{array}$ \\
\hline blade mass & $\mathrm{kg}$ & 54,675 & $52,187(-4.5 \%)$ & $47,302(-13.5 \%)$ & $53,195(-2.7 \%)$ \\
\hline AEP & $\mathrm{MWh} / \mathrm{yr}$ & 23.48 & $23.42(-0.3 \%)$ & $23.29(-0.8 \%)$ & $23.62(+0.6 \%)$ \\
\hline $\mathrm{m} / \mathrm{AEP}$ & $\mathrm{kg} * \mathrm{yr} / \mathrm{MWh}$ & 0.2741 & $0.2738(-0.1 \%)$ & $0.2732(-0.3 \%)$ & $0.2720(-0.8 \%)$ \\
\hline
\end{tabular}

\section{RESULTS}

The results from the optimization are shown in Table III. For this analysis, we compare the results from three different optimization cases: the conventional case with the material and airfoil thicknesses fixed, only the airfoil thickness fixed, and the full optimization. The optimization results are all compared to the NREL 5-MW reference turbine. A comparison of the design variables of interest are shown in Fig. 6. These include the airfoil thickness to chord ratio, chord, twist, spar cap TRIAX thickness, spar cap carbon thickness, trailing edge foam thickness, and trailing edge TRIAX thickness.

The first optimization used the conventional approach with the airfoil and material layer thicknesses fixed. The optimization was able to reduce the blade mass substantially $(-4.5 \%)$, however, this also had the negative impact of reducing the energy production $(-0.3 \%)$. The main area in which the blade mass was reduced was through the chord decrease near the root. Farther along the blade span the chord increased slightly for an average chord decrease of $3.5 \%$. The twist decreased on average by $7.5 \%$ in an attempt to extract a better aerodynamic performance. These changes allowed the tip-speed ratio to increase by $5.7 \%$. This led to a very modest decrease in $m / A E P(-0.1 \%)$.

The second optimization added in the material layer thicknesses in the composite lamina section of both the spar cap and the trailing edge panels. The addition of these thicknesses led to a significantly smaller blade mass $(-13.5 \%)$. However, similar to the first optimization the benefit from reducing the blade mass was in part negated by the reduction in energy production $(-0.8 \%)$. The chord was also decreased in this case by about $3.1 \%$, slightly less than in the first optimization. The twist decreased more so in this second optimization by an average of $14.5 \%$. The tip-speed ratio increased by $7.5 \%$. The main difference in this second optimization was in the material layer thicknesses. In both the spar cap and the trailing edge panels the TRIAX layer thicknesses decreased significantly. On the other hand, the carbon and foam layers in the spar cap and the trailing edge increased respectively. This result shows that the conventional method of adding these layers as a single design variable is limiting the performance. As a single variable these thicknesses would have to either both increase or both decrease. We see, however, that in both cases the two layers moved in opposite directions with one increasing and the other decreasing. Another interesting effect was that the overall change in the total composite layer thickness increased, though by very different amounts for the spar cap $(+17.5 \%)$ and trailing edge $(+0.05 \%)$. In both cases, the thicknesses increased, which is interesting because the mass was significantly reduced. The spar cap thickness increased substantially while the trailing edge in essence did not change at all. The TRIAX layer is more dense than both the carbon and the foam layers and to reduce the mass the TRIAX layer thicknesses decreased and was replaced with either the carbon or foam layers. If the layers were combined into a single variable then this result would be impossible. The addition of material layer thicknesses further reduced the $m / A E P$ by $0.2 \%$ (from $-0.1 \%$ to $-0.3 \%$ ).

For the third and final optimization, the airfoil thickness to chord ratios were added and caused several interesting results. One of the main results was the change in blade mass and $A E P$. The blade mass decreased $(-2.7 \%)$, but not nearly as much as in the first two cases. However, unlike the other cases the energy production in this case actually increased by a fair margin $(+0.6 \%)$. By adding $t / c$ the blade was able to both reduce mass as well as increase 


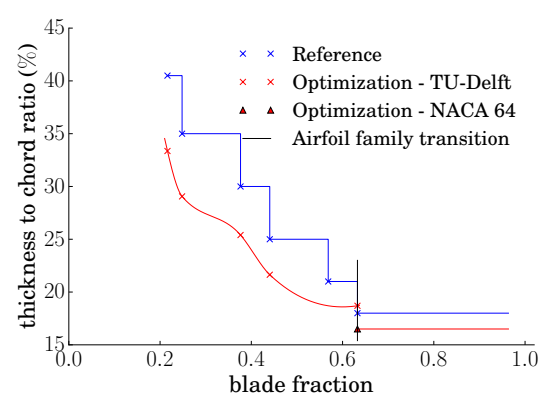

(a) Airfoil thickness to chord ratio

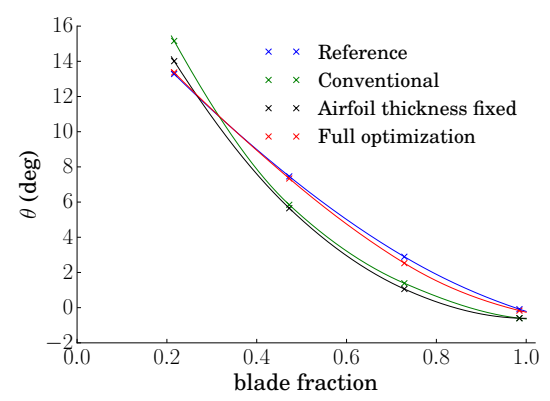

(c) Twist

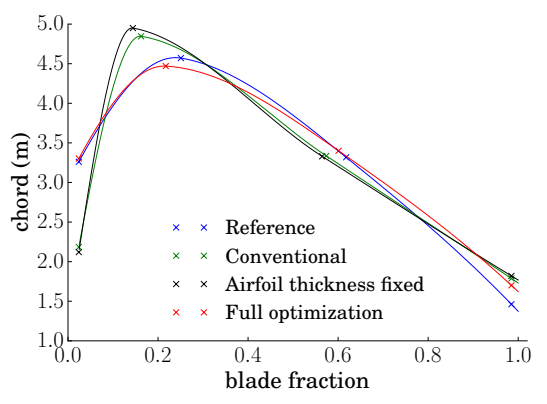

(b) Chord

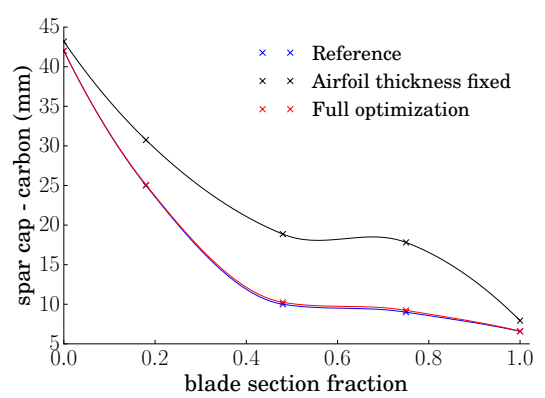

(d) Spar cap - carbon thickness

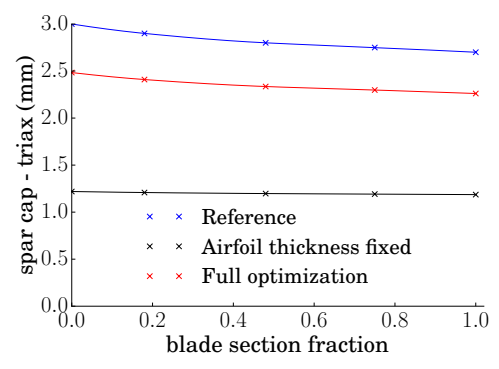

(e) Spar cap - TRIAX thickness

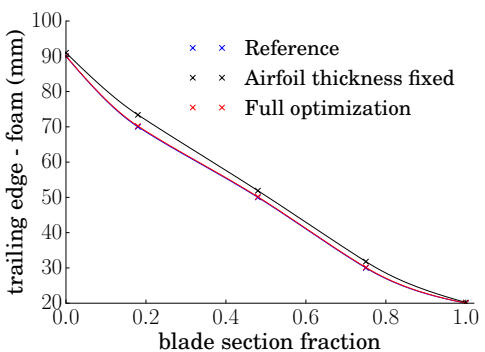

(f) Trailing edge - foam thickness

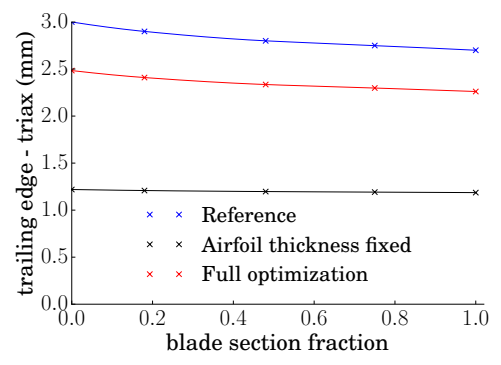

(g) Trailing edge - TRIAX thickness

Fig. 6: Comparison of results between the various optimization cases

energy production. Another interesting result is that the design variables were much closer to the reference blade than in the other optimization cases. The chord actually increased by an average of $2.1 \%$ as opposed to where it decreased in the other cases. The twist only decreased by $2.0 \%$. The tip-speed ratio increased by $2.6 \%$. The addition of the airfoil thicknesses actually made the majority of the design variables more closely match the reference blade. In addition, the material thicknesses decreased in the spar cap $(-1.6 \%)$ and the trailing edge $(-0.6 \%)$ panels while in the other cases the material layer thicknesses increased, Since the chord increased, the major source of blade mass reduction was in decreasing the airfoil thicknesses for an overall thinner blade. The average airfoil thickness reduction was significant at $18.5 \%$. Therefore, a thinner blade with a thicker chord was better than a smaller chord with a thicker blade. The addition of $t / c$ further reduced the $m / A E P$ by $0.5 \%$ (from $-0.3 \%$ to $-0.8 \%$ ).

There are several important results that are obtained from this research. Material layer thicknesses in the composite lamina section should be separated during the optimization process. The two different material layer thicknesses in both the spar cap and the trailing edge panels went in opposite directions, which is not possible when only using one design variable. A substantial gain can be obtained by adding airfoil thicknesses. The biggest benefit is not in mass reduction but in improved energy production. The thinner airfoils have better aerodynamic performance and are able to extract more energy from the wind. Adding all of the 
design variables together had improved results over the sequential design with the airfoils fixed and resulted in very different blade designs. The trade-offs between the aerodynamic performance of the blade and the thicknesses of the airfoil, composite lamina layers, and chord length are better explored by the optimization as a result. The further reduction of $0.5 \%$ when adding the airfoil thickness is similar to the reduction of $0.3 \%$ (from a $C O E$ of $-1.8 \%$ to $-2.1 \%$ ) obtained by Bottasso et al. in their free-form approach of $C O E$ [2]. Although different methods and conditions were used between this research and their free-form approach, the comparable results show the potential of using airfoil thickness to capture part of the benefits from the free-form approach. In all three optimization cases, the tip-speed ratio increased. This alludes to the idea that this method could be applied successfully to high tip-speed turbines. Increased saving at high tip-speeds would be likely when incorporating the entire tower instead of just the rotor as the reduction of the drivetrain mass, which is the major source of cost reduction for high tip-speed turbines, is not included in this analysis.

Although the percent improvement was modest, adding airfoil thickness and material layer thicknesses could have an important contribution in making wind energy a more appealing energy source through lower costs and higher energy production.

\section{CONCLUSION}

From this analysis, there is an increased ability to compare the trade-offs between the aerodynamic and structural design of wind turbine blades The results show the potential of both separating the material layer thicknesses and adding $t / c$ for improved wind turbine blade performance. Additional work includes a full free-form design that determines the differences between $t / c$ as well as explores additional benefits of the free-form approach. The further decrease of $0.5 \%$ $(-0.3 \%$ to $-0.8 \%)$ by adding airfoil thickness and $0.2 \%$ $(-0.1 \%$ to $-0.3 \%)$ by adding material layer thicknesses show the benefit to be gained from integrating this research into conventional blade optimization. It is recommended, where applicable, that $t / c$ and the material layer thicknesses be added as design variables to wind turbine blade optimization so as to further enhance the appeal of wind energy as a viable alternative energy source.

Future work will be important in continuing to develop the results. Additional performance and higher fidelity results are likely to be obtained by using a computational fluid dynamics model instead of XFOIL. XFOIL is a good preliminary tool to show the feasibility of the results, however, better data can be obtained through higher fidelity tools. Additional structural materials, such as a material thickness distribution for the structural web, could further improve the results. A complete free-form design would increase blade performance and the results compared to this research. Additional work, including investigations into high tip-speed turbines and a full cost of energy analysis, could be performed to further increase the applicability and fidelity of these results.

\section{REFERENCES}

[1] K.-N. Chen and P.-Y. Chen, "Structural optimization of 3 MW wind turbine blades using a two-step procedure," Int. J. Simul. Multidisci. Des. Optim., vol. 4, no. 3, 2010.

[2] C. L. Bottasso, A. Croce, and L. Sartori, "Free-form design of low induction rotors," AIAA SciTech, 2015.

[3] K. Dykes, A. Ning, R. King, P. Graf, G. Scott, and P. Veers, "Sensitivity analysis of wind plant performance to key turbine design parameters: A systems engineering approach," in 32nd ASME Wind Energy Symposium, National Harbor, Maryland, January 2014.

[4] A. Ning and K. Dykes, "Understanding the benefits and limitations of increasing maximum rotor tip speed for utility-scale wind turbines," Journal of Physics: Conference Series, vol. 524, no. 012087, June 2014.

[5] REUK, "Wind turbine tip speed ratio." [Online]. Available: http://www.reuk.co.uk/Wind-Turbine-Tip-Speed-Ratio.htm

[6] G. K. W. Kenway and J. R. R. A. Martins, "Aerostructural shape optimization of wind turbine blades considering site-specific winds," in Proceedings of the 12th AIAA/ISSMO Multidisciplinary Analysis and Optimization Conference, Victoria, BC, September 2008.

[7] A. Ning, R. Damiani, and P. J. Moriarty, "Objectives and constraints for wind turbine optimization," Journal of Solar Energy Engineering, vol. 136, no. 4, pp. 04 010-041 010, June 2014.

[8] M. Drela, "XFOIL: An analysis and design system for low reynolds number airfoils," in Low Reynolds Number Aerodynamics, ser. Lecture Notes in Engineering, T. Mueller, Ed. Springer Berlin Heidelberg, 1989, vol. 54, pp. 1-12.

[9] A. Ning, "NREL WISDEM Airfoilpreppy." [Online]. Available: http://wisdem.github.io/AirfoilPreppy

[10] — "A simple solution method for the blade element momentum equations with guaranteed convergence," Wind Energy, vol. 17, no. 9, pp. 1327-1345, 2014.

[11] — "NREL WISDEM RotorSE." [Online]. Available: http://wisdem.github.io/RotorSE/

[12] European Committee for Standardisation, "Eurocode 3: Design of steel structures-part 1-6: General rules-supplementary rules for the shell structures," European Committee for Standardisation, EN 1993-1-6: 20xx, 1993.

[13] G. S. Bir, User's guide to PreComp (Pre-Processor for Computing Composite Blade Properties). National Renewable Energy Laboratory Golden, Colo, 2006.

[14] P. E. Gill, W. Murray, and M. A. Saunders, "SNOPT: An SQP algorithm for large-scale constrained optimization," SIAM Journal on Optimization, vol. 12, no. 4, pp. 979-1006, 2002.

[15] J. S. Gray, K. T. Moore, and B. A. Naylor, "OpenMDAO: An Open Source Framework for Multidisciplinary Analysis and Optimization," in 13th AIAA/ISSMO Multidisciplinary Analysis and Optimization Conference, Fort Worth, TX, AIAA, AIAA2010-9101. Fort Worth, Texas: AIAA, August 2010. 